\title{
On the possibility of extending the IGRF predictive secular variation model to a higher SH degree
}

\author{
Luís Silva ${ }^{1}$, Stefan Maus ${ }^{2}$, Gauthier Hulot ${ }^{1}$, and Erwan Thébault ${ }^{1}$ \\ ${ }^{1}$ Equipe de Géomagnétisme, Institut de Physique du Globe de Paris, Université Paris Diderot, INSU/CNRS, \\ 4, Place Jussieu, 75252, Paris, cedex 05, France \\ ${ }^{2}$ National Geophysical Data Center, NOAA E/GC1, 325 Broadway, Boulder, CO 80305-3328, USA
}

(Received January 22, 2010; Revised June 14, 2010; Accepted July 1, 2010; Online published December 31, 2010)

\begin{abstract}
The International Geomagnetic Reference Field (IGRF) is an internationally agreed global spherical harmonic model of the Earth's magnetic field of internal origin. It is currently computed every five years in the form of a model describing this field up to degree 13 at a reference epoch, plus a secular variation model up to degree 8, best estimating the linear evolution of this field over the following five years. Such a simple description of the field evolution is thought to provide a good enough prediction of the field, both for navigational and internationally agreed reference purposes (the very purpose of IGRF models). In particular, it assumes that any change in the field described by spherical harmonic degrees between 9 and 13 may be neglected over five years, given the uncertainties already involved in the determination of all other coefficients, and the practical accuracy needed for most IGRF applications. Recent progress in global field modelling based on increasingly accurate and numerous satellite data however show that all field coefficients can now be computed with much higher accuracy than possible in the past, and that higher degree secular variation coefficients could therefore also be considered for inclusion in IGRF models. The present short note intends to investigate the potential benefit of extending the IGRF predictive secular variation model to degrees higher than 8 , given our current knowledge of the way the field behaves over time periods of five years.
\end{abstract}

Key words: Geomagnetism, field modelling, reference field, secular variation.

\section{Introduction}

The International Geomagnetic Reference Field (IGRF) is a reference mathematical model of the Earth's main magnetic field, presented in terms of Gauss coefficients up to degree and order 13 and their predictive first time derivatives up to degree and order 8 for the upcoming five years. It is released by the International Association of Geomagnetism and Aeronomy (IAGA) and results from the collaboration effort between magnetic field modellers and the institutes that collect and make magnetic field data available. Its recent release, IGRF-11 (Finlay et al., 2010) comprises retrospectively-produced models for previous epochs from 1900 to 2005 (definitive 1945-2005), an estimate of the main field in 2010 up to spherical harmonic (SH) degree and order 13 and a predictive secular variation up to $\mathrm{SH}$ degree and order 8 with predictive value until 2015. Thus, the predictive secular variation does not include the smaller-scale evolution of the field between degrees 9 and 13 .

By construction, the IGRF model is defined as a weighted average of candidate models submitted for evaluation by various teams (Finlay et al., 2010). There is no agreement on how to derive the optimum secular variation model and IGRF candidates generally follow rather different methodologies. Classically, the secular variation computed as the

Copyright (C) The Society of Geomagnetism and Earth, Planetary and Space Sciences (SGEPSS); The Seismological Society of Japan; The Volcanological Society of Japan; The Geodetic Society of Japan; The Japanese Society for Planetary Sciences; TERRAPUB.

doi:10.5047/eps.2010.07.005 average of the main field time derivative over the previous five years is considered to approximate well the secular variation over the next five years (Beggan and Whaler, 2010). More instantaneous estimates may nevertheless be proposed. They are obtained from finite difference of data extrapolated at different future epochs (Chambodut et al., 2010), from time-varying models extrapolated to the epoch of the main field IGRF model (e.g., Maus et al., 2010; Olsen et al., 2010; Thébault et al., 2010) or to future epochs (Lesur et al., 2010). In this short note however, we focus on the interest of computing predictive secular variation models as the average rate of change of the main field over the previous five years as given by retrospective models.

Recent field models such as POMME-3.0 (Maus et al., 2006), CHAOS (Olsen et al., 2006), xCHAOS (Olsen and Mandea, 2008), GRIMM (Lesur et al., 2008) or CHAOS-2 (Olsen et al., 2009), consider an instantaneous internal field up to $\mathrm{SH}$ degree 13 or higher and a temporal expansion of the Gauss coefficients made in terms of some set of functions (degree 2 polynomials for POMME-3.0, cubic Bsplines for CHAOS and xCHAOS, order $5 \mathrm{~B}$-splines for GRIMM and CHAOS-2). As a result, these models also consider an instantaneous secular variation up to at least degree 13 and a second time derivative considered robust up to degree 5 or 6 . The availability of these high quality retrospective field models provides the basis and stimulus for extending the limits of any operational model such as IGRF towards more spatially accurate short-term predictions. 
Here we thus consider the potential benefits of improving IGRF's predictive power by increasing the SH degree of its predictive secular variation. We will show that these high degree coefficients correlate reasonably well over time, are quite well determined and have potential predictive value over the five years validity period of IGRF.

\section{Correlation between Average Secular Variation Estimates from Published Models}

We first briefly assess the extent to which average secular variation models computed over some period of time before a reference epoch correlate with an average secular variation computed over 5 years after this reference epoch. For that purpose, we make use of the CM4 model of Sabaka et al. (2004) over the time period going from 1980 to 1999 , and of the CHAOS-2 model of Olsen et al. (2009) over the time period going from 1999 to 2009 (more specifically, its smooth version CHAOS-2s), and compute the following mean secular variations:

SV1 the mean secular variation between 1980 and 1999, by taking the difference between the field at 1980 and 1999 from CM4;

SV2 the mean secular variation between 1994 and 1999, by taking the difference between the field at 1994 and 1999, again from CM4;

SV3 the mean secular variation between 1999 and 2004, still computed in the same way but from CHAOS-2s;

SV4 the mean secular variation between 2004 and 2009, again computed from CHAOS-2s;

We next define the degree-by-degree correlation of two magnetic field models over the surface of a sphere as a function of the Gauss coefficients of their expansion in terms of SH. For each degree we have (e.g. Langel and Hinze, 1998, section 4.5.2):

$$
C_{n}=\frac{\sum_{m=-n}^{n} g_{n}^{m}(1) g_{n}^{m}(2)}{\sqrt{\left(\sum_{m=-n}^{n} g_{n}^{m}(1)^{2}\right) \times\left(\sum_{m=-n}^{n} g_{n}^{m}(2)^{2}\right)}}
$$

where (1) and (2) specify the model from which the Gauss coefficients are taken and $g_{n}^{m}$ for negative $m$ are given by $g_{n}^{m}=h_{n}^{|m|}$. The correlation $C_{n}$ between secular variation models can be computed in the same way by just replacing the Gauss coefficients $g_{n}^{m}$ by their first time derivatives $\dot{g}_{n}^{m}$ where we adopted the "dot" notation for time derivatives. We shall consider a correlation to be good for all values over 0.8 .

We first consider 1999 as our reference epoch, and compute the per degree correlation between either of the two average SV1 (1980-1999) and SV2 (1994-1999) secular variation models computed from $\mathrm{CM} 4$, and the average SV3 (1999-2004) secular variation model computed from CHAOS-2s (Fig. 1).

This first calculation shows that a better correlation is achieved when considering an average secular variation computed over a five year time period (SV2) before the reference epoch, than when considering an average secu-

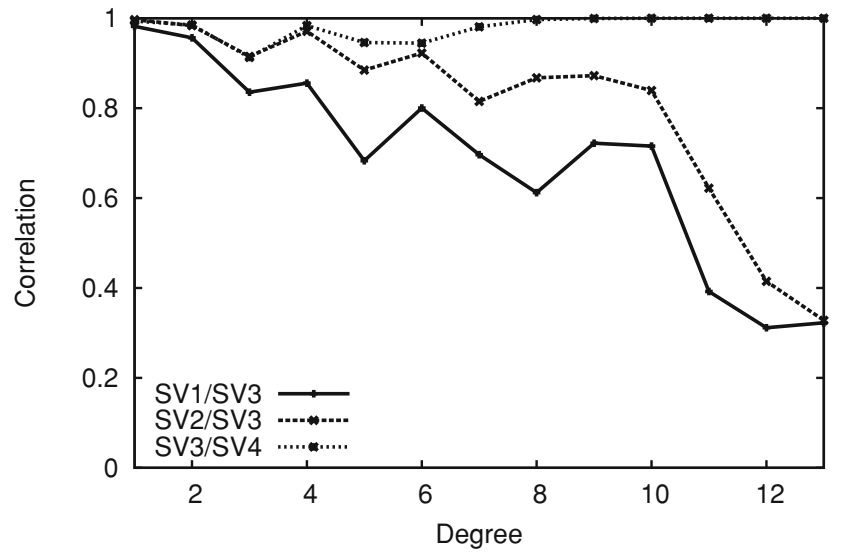

Fig. 1. Per degree correlations between mean secular variations: solid line, SV1 (mean secular variation between 1980 and 1999) and SV3 (mean secular variation between 1999 and 2004); dashes, SV2 (mean secular variation between 1994 and 1999) and SV3; dots, SV3 and SV4 (mean secular variation between 2004 and 2009).

lar variation computed over a significantly longer time period (SV1). This is particularly true for the largest degrees which, as noted by one of the reviewers, can be related to the fact that higher degrees involve shorter time-scales (e.g. Hulot and Le Mouël, 1994). To confirm this first result, we also computed the per degree correlation between SV3 (1999-2004) and SV4 (2004-2009), both computed from CHAOS-2s, now considering 2004 as the reference epoch (Fig. 1). Again, and as expected, SV3 and SV4 appear to correlate very well.

The two SV2/SV3 and SV3/SV4 set of correlations shown in Fig. 1 are however well worth commenting a little further, as they not only provide information about the secular variation itself, but also strongly reflect the regularisation being used in the parent models CM4 and particularly CHAOS-2s. Whereas those two correlation sets roughly provide the same results up to degree 6 , the seemingly remarkable correlations between SV3 and SV4 from degree 7 to 13 , indeed simply reflect the fact that both SV3 and SV4 are derived from the same CHAOS-2s model, and that the time variations in this model are strongly regularised beyond degree 7 . The corresponding degrees of the secular acceleration over the 1999-2009 time period are indeed severely damped (see figure 3 in Olsen et al. (2009)) and, as a result, SV3 and SV4 are artificially forced to be close to identical for those degrees. A similar effect is likely to also affect $\mathrm{CM} 4$, and we must therefore be careful in drawing conclusions from Fig. 1. But an interesting message can still be inferred from the SV2/SV3 set of correlations. These secular variation models were computed from CM4 (SV2) and CHAOS-2s (SV3) which were built from essentially different data sets using different modelling methodologies. Even if the two data sets do slightly overlap over a 4.5 years period about the reference epoch 1999 (CM4 relies on data covering the 1960-2002.5 time period, whereas CHAOS-2s relies on data covering 1997-2009.5) they can be considered as essentially independent. The fact that the five year average secular variation models before (SV2) and after (SV3) the 1999 reference epoch show a correlation su- 
perior to 0.8 up to degree 10 can then be taken as a first indication that not only degrees 1 to 8 but also degrees 9 and 10 of a secular variation model computed over a period of 5 years could have some useful predictive power.

\section{Comparison between Main Field Forecasts}

A more direct way of testing the potential benefit of using degrees higher than 8 in IGRF secular variation models, is to simply compare the predicted field five years after the considered reference epoch, with a model directly supposed to properly reflect the field at that epoch. For that purpose, we extrapolated the $1999\left(t_{0}\right)$ main field of CHAOS-2s to $2004(t)$ by making use of a fraction of either SV1 and SV2 through the expression:

$$
g_{n}^{* m}(t)=g_{n}^{m}\left(t_{0}\right)+c \dot{g}_{n}^{m} \delta t
$$

where $g_{n}^{* m}(t)$ stands for the Gauss coefficients of the predicted field in 2004, $g_{n}^{m}\left(t_{0}\right)$ for those of CHAOS-2s in 1999, $\dot{g}_{n}^{m}$ for those of either SV1 or SV2, $c$ is a constant between 0 and 1 and $\delta t=t-t_{0}=5$ years. The constant $c$ was introduced in order to also measure the consequences of only considering a fraction, rather than all, of the mean secular variation to better assess its usefulness for the purpose of extrapolating the field over the next five years. Setting $c=0$ then is the same as having no secular variation acting during the given 5 year period.

We then compared those predictions of the 2004 main field with the values directly provided by CHAOS-2s for that epoch. The r.m.s. misfit associated with those predictions is defined by:

$$
\Delta \mathrm{B}_{n}(c)=\sqrt{(n+1) \sum_{m=-n}^{n}\left[g_{n}^{m}(t)-g_{n}^{* m}(t)\right]^{2}}
$$

where $g_{n}^{m}(t)$ stands for the Gauss coefficients provided by CHAOS-2s in 2004.

Then, for each degree $n$, the improvement brought by assuming some steady secular variation acting on the field during the $\delta t=5$ year period between $t_{0}=2004$ and $t=2009$, relatively to assuming no secular variation can be written as:

$$
I_{n}(c)=\frac{\Delta \mathrm{B}_{n}(0)-\Delta \mathrm{B}_{n}(c)}{\Delta \mathrm{B}_{n}(0)} \times 100
$$

Having a $100 \%$ relative improvement then means that, by making use of the given secular variation, we properly predicted the field at the end of the epoch. By contrast, a relative improvement of $0 \%$ means that the secular variation was of no use.

This relative improvement, for the Gauss coefficients taken at the Earth's surface, is plotted in Fig. 2 when using SV1, and in Fig. 3 when using SV2, where each line corresponds to a different value of $c$. It is interesting to see that even SV1 which, we recall, is the mean secular variation of CM4 between 1980 and 1999, produces some improvement in the prediction of all degrees, including those above 8 .

Much more interesting, however, is the result obtained when considering SV2 (Fig. 3), which now suggests a

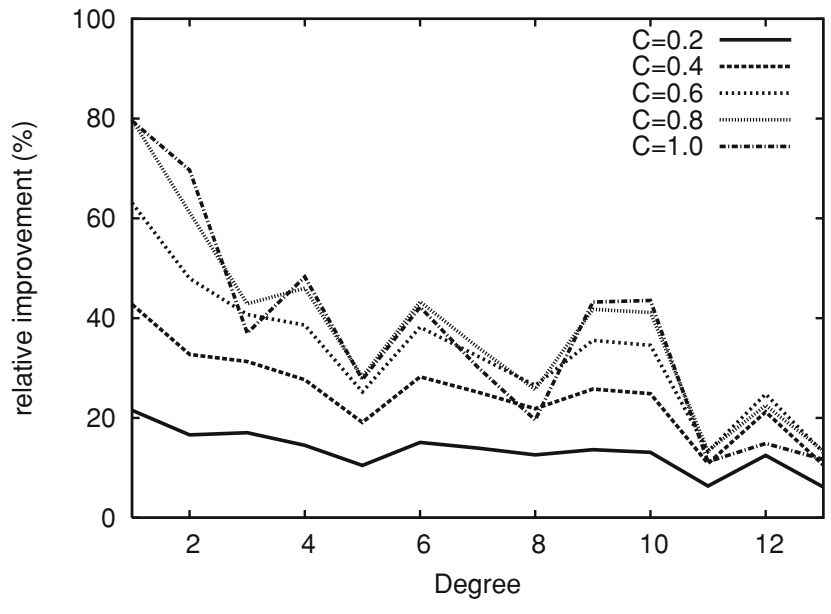

Fig. 2. Per degree relative improvement in misfits between the CHAOS-2s 1999 main field model extrapolated to 2004 using the SV1 secular variation model, and the CHAOS-2s 2004 main field model (see text for details).

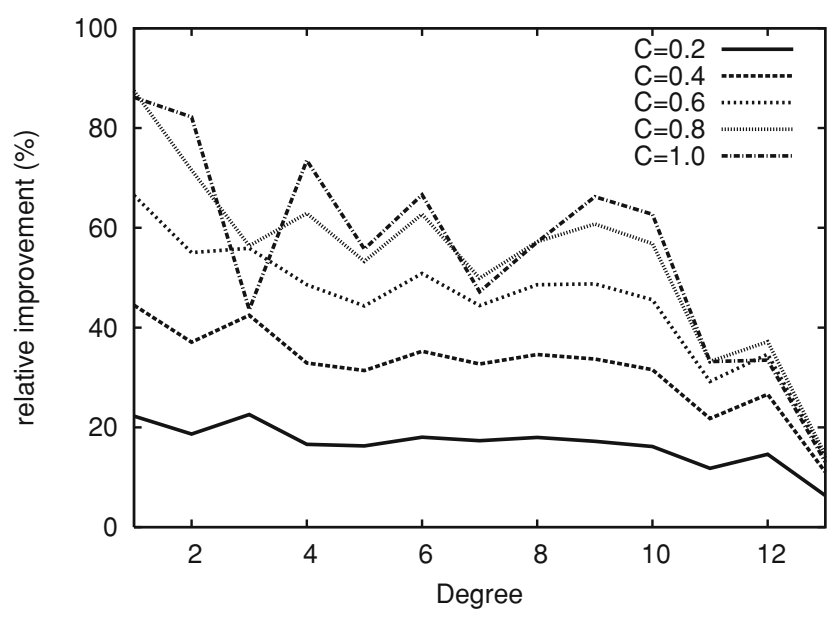

Fig. 3. Per degree relative improvement in misfits between the CHAOS-2s 1999 main field model extrapolated to 2004 using the SV2 secular variation model, and the CHAOS-2s 2004 main field model (see text for details).

strong benefit of using this model up to degree 12 . Degrees 9 and 10 are now particularly interesting as they show a relative improvement well above $60 \%$, comparable to that for degrees up to 8 and in fact higher than for degrees 3 and 7. Again, this suggests that considering a predictive secular variation up to at least degrees 9 and 10 constitutes a real improvement when comparing to the present static field approach of IGRF. Infact, Fig. 3 again suggests that considering degrees 11 and 12 is also of some potential benefit, although the relative improvement in the misfit is clearly inferior.

Finally, note that, as we in fact also checked, carrying out a similar calculation with SV3, and using 2004 and 2009 from CHAOS-2s as initial and final reference field models, does not bring any useful additional information, as SV3 is also directly inferred from CHAOS-2s, leading the results to mainly reflect the temporal regularisation used in CHAOS-2s, which we already discussed in the previous section. 


\section{Testing Predictions from a Degree 13 Extended- IGRF-10 Secular Variation Model against a Fully Independent Model}

There are two main limitations to the tests considered so far. First, all tested secular variation models were derived from parent models in a way that is not necessarily representative of the way IGRF secular variation models are constructed. Second, all comparisons were made with control models (be it SV3, SV4 or CHAOS-2s for epoch 2004), that are not fully independent from the tested secular variation models. In particular, because CHAOS-2s was built from data covering the 1997-2009.5 time period, with some amount of temporal regularisation, it may rightly be objected that its estimate of the 2004 field may be favourably biased towards the prediction inferred from SV1 and SV2, both based on CM4, which considered data up to 2002.5. In order to complement the tests already reported, we therefore decided to perform a final set of tests that avoid those limitations.

We first produced an extended version of the IGRF-10 (Maus et al., 2005) predictive secular variation model using the same kind of methodology as the one used for the published version, but extended up to degree 13. This model we refer to as EIGRF. It was constructed with the help of models Ørsted(09d/04) and Ørsted(09g/04) of Olsen et al. (2005) and POMME-3.0 of Maus et al. (2006), all of which contributed to IGRF-10 or came directly from IGRF-10 parent models and are freely available. The coefficients of EIGRF, which are secular variation coefficients, we recall, were computed as the simple average of the mean secular variation between epoch 2000 and epoch 2005 of the $N=3$ above-mentioned models. That is:

$$
\left\langle\dot{g}_{n}^{m}\right\rangle=\frac{1}{5 N} \sum_{i=1}^{N}\left(g_{n}^{m(i)}(2005)-g_{n}^{m(i)}(2000)\right)
$$

where $i$ iterates over the $N=3$ models.

Figure 4 shows the power spectrum (Lowes, 1974) of this new secular variation model at the Earth's surface, together with that of the published IGRF-10 predictive secular variation model and of the difference between the two. We can see that for SH degree 8 and below our EIGRF secular variation model is very similar to that of IGRF-10. It thus seems reasonable to assume that this EIGRF model is representative of the predictive secular variation that would have been published as IGRF-10, had the request been made that it should extend to degree 13 .

To test and compare the predictive power of both IGRF10 and EIGRF, we next derived a magnetic field model, similar in construction to the POMME-3.0 model (Maus et al., 2006) and to the POMME-5 model (which can be found at http://geomag.org/models/pomme5.html) but only constructed from post-2005.0 data, and valid for the period going from 2005.0 through mid 2009. This model we refer to as POMME5-post05. It features a quadratic development of the Gauss coefficients in time, centred around 2007.0 and built strictly from CHAMP data pertaining to epochs between 2005.0 and 2009.3 (see Table 1). The external field parametrisation and coefficients are identical to those used for POMME-3.0. At low latitudes, only satellite tracks ver-

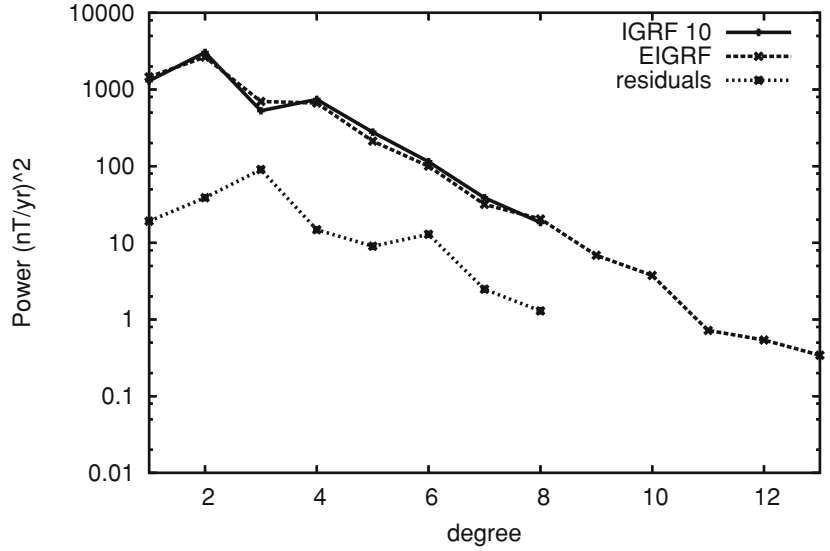

Fig. 4. Power spectra of the degree 8 IGRF-10 predictive secular variation model (solid) and of the degree 13 EIGRF secular variation model (dashes). Also shown (dots) is the power spectrum of the difference between these two models. All spectra are plotted at Earth's surface.

Table 1. Data used to construct POMME5-post05 according to location. Mid latitudes refer to track segments covering $-60^{\circ}$ to $60^{\circ}$ geomagnetic latitude (Mlat) and high latitudes refer to overlapping tracks at $<-50^{\circ}$ and $>50^{\circ}$ (Mlat).

\begin{tabular}{l|c} 
& $n$. data used \\
\hline Scalar data (mid latitude) & 358345 \\
Scalar data (north polar) & 239928 \\
Scalar data (south polar) & 235503 \\
Vector data (mid latitude) & 252879
\end{tabular}

ified as being undisturbed by ionospheric plasma irregularities were used (Stolle et al., 2006) and the input data were corrected for the magnetic signal of ocean tidal induction. By construction, this model is thus completely independent of IGRF-10 and EIGRF. For that reason, it can be considered as a satisfying model of the field for comparisons with IGRF-10 and EIGRF based field forecasts throughout its period of validity.

It is instructive to first investigate how the predictive EIGRF secular variation model compares with the secular variation of POMME5-post05 between 2005 and 2009. This is shown in Fig. 5 in the form of per degree correlations at each year of that time interval. The highest correlations are obtained when comparing the EIGRF secular variation with the POMME5-post05 secular variation in 2005 up to and including $\mathrm{SH}$ degree 9 . The correlation progressively decreases (roughly by the same amount for all degrees between 5 and 9) when considering more recent epochs. By 2009 , correlations for degrees between 5 and 10 are very similar and it is very encouraging to see that correlations for even higher degrees are still quite significant. They are even somewhat more significant than when we compared SV2 and SV3 in Fig. 1.

Epoch 2009 is particularly interesting as it is near the limit of validity of the IGRF's predictions and allows us to test, not only the predictive power of IGRF's secular variation but also that of EIGRF. Direct comparison between the predictive EIGRF secular variation model and the secular variation of POMME5-post05 in 2009 can be made by plotting the power spectrum of the difference between them, and comparing it to the power spectrum of the latter. This is 


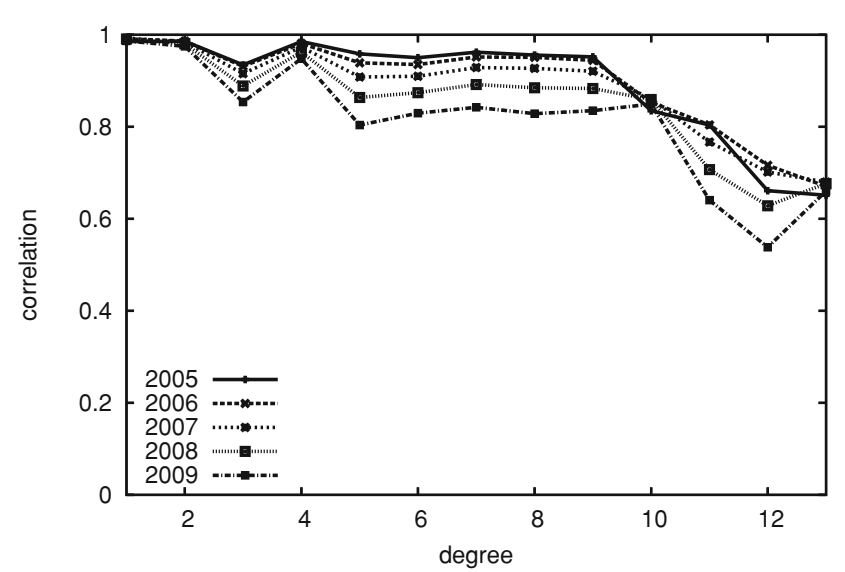

Fig. 5. Per degree correlations between the EIGRF secular variation model and the POMME5-post05 secular variation in 2005, 2006, 2007, 2008 and 2009 .

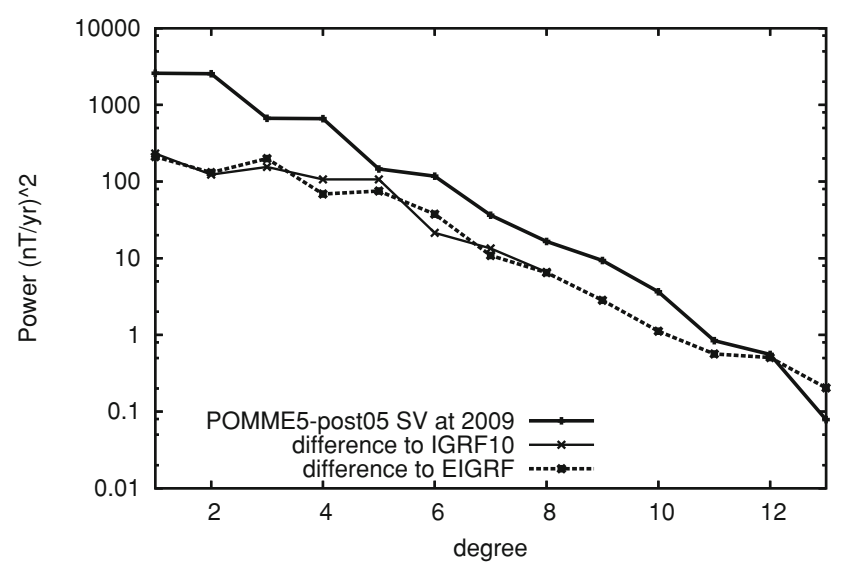

Fig. 6. Power spectra of the POMME5-post05 2009 secular variation model (solid thick line) and of the differences between this model and the IGRF-10 predictive secular variation model (up to degree 8, solid thin line) or the EIGRF predictive secular variation model (up to degree 13, dashes). All spectra are computed at Earth's surface.

shown in Fig. 6, where the power spectrum of the difference between the published IGRF-10 predictive secular variation model and the POMME5-post05 2009 secular variation model is also shown. We can see that EIGRF provides an estimate of the 2009 secular variation that is as good as the one provided by IGRF-10 up to degree 8 . Furthermore, the relative misfit between EIGRF and the POMME5-post05 2009 secular variation remains constant for degrees 9 and 10. The approximation is clearly not as good for degree 11 and the errors seem to dominate degrees 12 and 13 .

These results again strongly suggest that the high degrees of the secular variation (at least for degrees 9 and 10), computed as a mean over the preceding 5 year period, were sufficiently well resolved and correlated with the true secular variation to already have been included in IGRF-10.

Finally, we compared two predictions of the field at 2009 with the field as given by POMME5-post05 at that epoch. Both predictions were made using Eq. (2) with $\delta t=4$ years and $c=1$ where $g_{n}^{m}\left(t_{0}\right)$ now stands for the 2005 IGRF-10 field coefficients and $\dot{g}_{n}^{m}$ for the secular variation model coefficients given by either IGRF-10 up to degree 8 (first

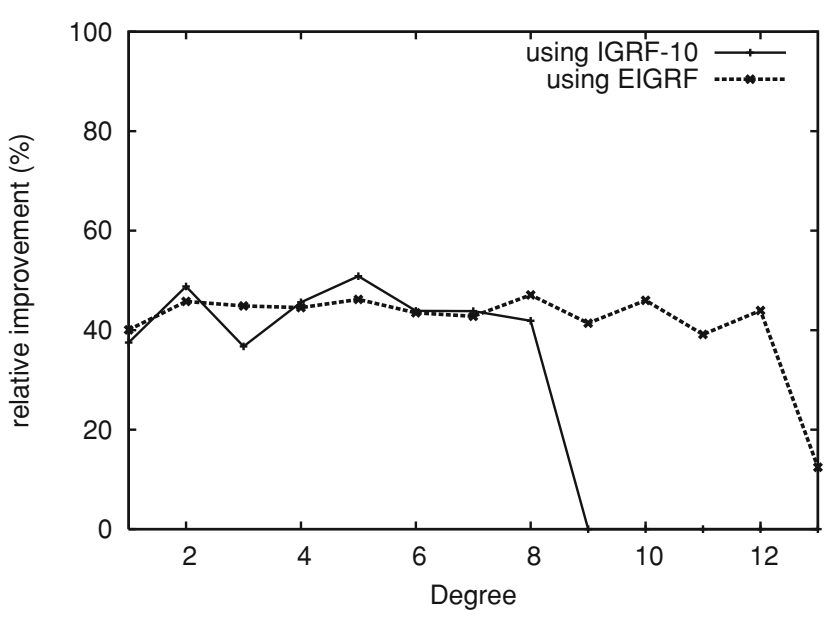

Fig. 7. Per degree relative improvement of the misfit between the 2009 POMME5-post05 main field and: the 2009 extrapolation of IGRF-10 when using its own predictive secular variation (solid line); the 2009 extrapolation of IGRF-10 when using EIGRF (dashed line); when using the field provided by IGRF-10 for 2005 as a reference.

prediction) or EIGRF up to degree 13 (second prediction). The relative improvement (as defined by Eq. (3) and (4) where $g_{n}^{m}(t)$ now stands for the POMME5-post05 model coefficients in 2009) associated with these two predictions when compared to the POMME5-post05 main field in 2009 are plotted in Fig. 7. This figure, analogous to Figs. 2 and 3 is again encouraging. It indeed now shows that the benefit of considering secular variation degrees above 8 over that period of time, is the same in relative terms as the benefit of considering the IGRF-mandatory secular variation degrees 1 to 8 , not only for degrees 9 and 10 , but also for degrees 11 and 12 . Only degree 13 fails to bring any improvement, a result consistent with those of Fig. 6.

\section{Conclusion}

The present study clearly suggests that considering degrees above 8 for inclusion in an IGRF type of predictive secular variation model could be of potential use, provided such a model is based on an estimate of the past secular variation over a short enough period of time, typically 5 years, as is usually the case for standard IGRF predictive secular variation models. This benefit is best expressed in relative terms per degree of the field it would predict (Fig. 7), in which case each degree up to degree 12 appears to bring as much benefit as degrees up to 8 , already included in standard IGRF predictive secular variation models.

Our results also suggest that, at least over the time period 2005-2009 we considered, degrees 9 and 10 of such an extended IGRF predictive secular variation would correlate with secular variation models computed a posteriori from independent data, just as well as degrees 5 to 8 (Figs. 1 and 5).

From these results we conclude that at least degrees 9 and 10, if not also degrees 11 and 12, of an IGRF type of predictive secular variation model, would have as good a predictive power as the first eight degrees already included in standard IGRF predictive secular variation models. From this perspective, we thus see no objective reasons to restrict 
this model to degree 8 rather than degree 10 or even degree 12.

It is nevertheless important to recognise that the improvement brought by the inclusion of higher degrees in IGRF predictive secular variation models would mainly be in relative terms per degree of the field, and would not compensate for the absolute errors entailed by the uncertainties with which this secular variation can be constructed up to degree 8. The total power accounted for by degrees above 8 is indeed small when compared to the errors associated to the lower degrees (recall Fig. 4). But we also note that even in absolute terms, errors associated with neglecting degrees 9 and 10 in an IGRF predictive secular SV would still be larger than the error produced by the uncertainties associated with degrees 7 and 8 already included in such models (again, recall Fig. 4). This, we believe, again pleads in favour of at least including degrees 9 and 10 in the construction of forthcoming IGRF predictive secular variation models.

It is finally important to keep in mind that all of the results derived above rely on the availability of high-quality data provided by on-going satellite missions. Should such satellite data suddenly fail to be available for some significant fraction of the five years time between two successive IGRF models, the situation would clearly be different. From this perspective, keeping the maximum degree of IGRF predictive secular variation models to its present value would definitely avoid having to adjust this degree to the quality of the available data every time a new IGRF model is to be produced. This concern is a significant practical one, given the operational purpose of IGRF models. But it is not one based on scientific grounds. In fact, it is well worth noting that a similar issue would anyway also affect the maximum degree of the IGRF main field, currently set to degree 13 , but which used to be set to degree 10 before continuous magnetic measurements from space became available. Since the continuous (or possibly near-continuous, in case of a slight delay) availability of such measurements, with improved quality, should be ensured with the soon to be launched Swarm mission (Friis-Christensen et al., 2006, 2009), it is our opinion that increasing the maximum degree of the IGRF predictive secular variation model to take advantage of the benefit it would bring should seriously be considered, especially if the retrospective analysis of the next IGRF-11 set of models confirms that the provision of non-zero secular variation coefficients would have resulted in an improved prediction all the way up to degree 12 or even 13.

Acknowledgments. The authors would like to thank the reviewers B. Langlais and I. Wardinski for their constructive reviews and the guest editor Chris Finlay for his most helpful comments. This work was partly funded by CNES. This is IPGP contribution number 3042 .

\section{References}

Beggan, C. and K. Whaler, Forecasting secular variation using core flows, Earth Planets Space, 62, this issue, 821-828, 2010.

Chambodut, A., B. Langlais, M. Menvielle, E. Thébault, A. Chulliat, and G. Hulot, Candidate models for the IGRF-11th generation making use of extrapolated observatory data, Earth Planets Space, 62, this issue, 745-751, 2010.

Finlay, C. C., S. Maus, C. D. Beggan, M. Hamoudi, F. J. Lowes, N. Olsen, and E. Thébault, Evaluation of candidate geomagnetic field models for IGRF-11, Earth Planets Space, 62, this issue, 787-804, 2010.

Friis-Christensen, E., H. Lühr, and G. Hulot, SWARM: A constellation to study the Earth's magnetic field, Earth Planets Space, 58(4), 351-358, 2006.

Friis-Christensen, E., H. Lühr, G. Hulot, R. Haagmans, and M. Purucker, Geomagnetic research from space, EOS, 90(25), 213-214, 2009.

Hulot, G. and J.-L. Le Mouël, A statistical approach to the Earth's main magnetic field, Phys. Earth Planet. Inter., 82, 167-183, 1994.

Langel, R. A. and W. Hinze, The Magnetic Field of the Earth's Lithosphere: The Satellite Perspective, Cambridge University Press, 1998.

Lesur, V., I. Wardinski, M. Rother, and M. Mandea, GRIMM: the GFZ Reference Internal Magnetic Model based on vector satellite and observatory data, Geophys. J. Int., 173(2), 382-394, 2008.

Lesur, V., I. Wardinski, M. Hamoudi, and M. Rother, The second generation of the GFZ Reference Internal Magnetic Model: GRIMM-2, Earth Planets Space, 62, this issue, 765-773, 2010.

Lowes, F., Spatial power spectrum of the main geomagnetic field and extrapolation to the core, Geophys. J. R. Astron. Soc., 36, 717-730, 1974.

Maus, S., S. Macmillan, T. Chernova, S. Choi, D. Dater, V. Golovkov, V. Lesur, F. Lowes, H. Lühr, W. Mai, S. McLean, N. Olsen, M. Rother, T. Sabaka, A. Thomson, and T. Zvereva, The 10th-Generation International Geomagnetic Reference Field, Geophys. J. Int., 161, 561-565, 2005.

Maus, S., M. Rother, C. Stolle, W. Mai, S. Choi, H. Lühr, D. Cooke, and C. Roth, Third generation of the Potsdam Magnetic Model of the Earth (POMME), Geochem. Geophys. Geosyst., 7(7), Q07008, 2006.

Maus, S., C. Manoj, J. Rauberg, I. Michaelis, and H. Lühr, NOAA/NGDC candidate models for the 11th generation International Geomagnetic Reference Field and the concurrent release of the 6th generation Pomme magnetic model, Earth Planets Space, 62, this issue, 729-735, 2010.

Olsen, N. and M. Mandea, Rapidly changing flows in the Earth's core, Nature Geosci., 1, 390-394, 2008.

Olsen, N., T. Sabaka, and F. Lowes, New parametrisation of external and induced fields in geomagnetic field modeling, and a candidate model for IGRF 2005, Earth Planets Space, 57, 1141-1149, 2005.

Olsen, N., H. Lühr, T. Sabaka, M. Mandea, M. Rother, L. Tøffner-Clausen, and S. Choi, CHAOS - A model of Earth's magnetic field derived from CHAMP, Ørsted, and SAC-C magnetic satellite data, Geophys. J. Int., 166(1), 67-75, 2006.

Olsen, N., M. Mandea, T. Sabaka, and L. Tøffner-Clausen, CHAOS-2A Geomagnetic field model derived from one decade of continuous satellite data, Geophys. J. Int., 179, 1477-1487, 2009.

Olsen, N., M. Mandea, T. J. Sabaka, and L. Tøffner-Clausen, The CHAOS3 geomagnetic field model and candidates for the 11th generation IGRF, Earth Planets Space, 62, this issue, 719-727, 2010.

Sabaka, T., N. Olsen, and M. Purucker, Extending comprehensive models of the Earth's magnetic field with Ørsted and CHAMP data, Geophys. J. Int., 159, 521-547, 2004.

Stolle, C., H. Lühr, M. Rother, and G. Balasis, Magnetic signatures of equatorial spread F as observed by the CHAMP satellite, J. Geophys. Res., 111, A02304, 2006.

Thébault, E., A. Chulliat, S. Maus, G. Hulot, B. Langlais, A. Chambodut, and M. Menvielle, IGRF candidate models at times of rapid changes in core field acceleration, Earth Planets Space, 62, this issue, 753-763, 2010 .

L. Silva (e-mail: lacsilva@ipgp.fr), S. Maus, G. Hulot, and E. Thébault 